\title{
Los aportes de tres corrientes evolucionistas neoschumpterianas a la discusión sobre políticas de innovación
}

Gabriel Yoguel*

Florencia Barletta**

Mariano Pereira***

Recebido: 18/01/2017 Versão Revisada (Entregue): 28/03/2017 Aprovado: 13/07/2017

\section{RESUMEN}

Este artículo presenta en forma estilizada algunos elementos que podrían contribuir a una agenda de discusión sobre política de innovación en un sentido amplio que se deriva de tres corrientes evolucionistas neo-schumpeterianas que comparten una ontología similar pero se focalizan en ejes diferenciados. Estas corrientes son: sistemas de innovación, modelos de auto-organización y causación acumulativa. Los tres enfoques aportan ideas de gran poder explicativo en torno a la manera en la que se manifiesta la innovación y el modo de intervención orientado a favorecer su dinámica. Partiendo de la identificación de los elementos comunes y complementarios de estos enfoques, el artículo se propone establecer un diálogo entre esas corrientes para contribuir a la discusión de un marco analítico que permita entender la dinámica de la innovación en América Latina.

Palabras Clave | Sistemas de Innovación; Auto-organización; Causación Acumulativa; Política de Innovación

\section{Códigos JEL | O25; O30; O32}

* Interdisciplinario de Estudios en Ciencia, Tecnología e Innovación, Buenos Aires, Argentina. E-mail: gyoguel@ungs.edu.ar.

** Universidad Nacional de General Sarmiento, Buenos Aires, Argentina. E-mail: mfbarletta@gmail.com.

*** Universidad Nacional de General Sarmiento y Centro Interdisciplinario de Estudios en Ciencia, Tecnología e Innovación, Buenos Aires, Argentina. E-mail: lic.mpereira@gmail.com. 


\title{
The contributions of three Neoschumpeterian and Evolutionary approaches to the debate on innovation policies
}

\begin{abstract}
This article stylizes the conceptual ideas and proposals of industrial and technological policy derived from three neo-Schumpeterian evolutionary approaches: Innovation Systems, Selforganizing models and Cumulative Causation. These three approaches provide ideas with great explanatory power about the way innovation is generated and the modes of public intervention. We propose a dialogue among them based on the identification of the common and complementary elements of these approaches. This dialogue aims to contribute to the discussion of an appropriate analytical framework to understand the dynamics of innovation in Latin American countries.
\end{abstract}

KEYwORDS | Innovation Systems; Self-organization, Cumulative Causation; Innovation Policy

JEL CODES | O25; O30; O32 


\section{Introducción}

En los últimos años se fueron configurando consensos básicos acerca de que la generación de procesos de cambio estructural y desarrollo requería una adecuada intervención pública a través de políticas que promuevan la innovación (CEPAL, 2012). Este consenso se fue gestando desde diferentes enfoques que difieren tanto en el plano teórico como aplicado en relación a por qué y cómo intervenir.

Desde una posición más cercana a la ortodoxia, la intervención del Estado a través de políticas de innovación se justifica por la presencia de fallas de mercado (ARROW, 1962). Desde esta perspectiva se considera que debido a las características de bien público que asume el conocimiento, el gasto en $\mathrm{I}+\mathrm{D}$ resulta inferior al correspondiente a una situación de óptimo paretiano porque las empresas innovadoras no logran apropiarse completamente de los beneficios de estas inversiones.

En contraposición a esta literatura, el enfoque evolucionista neo-schumpeteriano plantea diferentes críticas a la intervención centrada en fallas de mercado cuando se estudia la política de innovación (DOSI, 1988; METCALFE, 1995; POSSAS, 1985; LEE, 2013; AROCENA; SUTZ, 2006; CASSIOLATO; LATRES, 2008; CIMOLI et al., 2011; LALL; TEUBAL, 1998). Estos autores argumentan que la ubicuidad de las fallas de mercado limita su practicidad como guía de política. Sostienen que la sub-inversión en I+D no se deriva de un problema de sub-óptimo paretiano sino del bajo nivel de capacidades construidas por las firmas e instituciones a lo largo de su sendero evolutivo. Desde esta perspectiva se plantea que ciertas fallas de mercado identificadas por el mainstream, como la información asimétrica, son inherentes a los procesos de innovación.

El objetivo del artículo es contribuir a este debate a partir de un marco analítico general de intervención en política de innovación que combina elementos de tres corrientes evolucionistas neoschumpeterianas identificadas en Robert y Yoguel (2016): sistemas de innovación (LUNDVALL, 1992; FREEMAN, 1987; NELSON, 1993; EDQUIST, 1997; LUNDVALL et al., 2009); auto-organización (METCALFE, 1995; DOPFER, FOSTER; POTTS, 2004); y causación acumulativa (MARENGO; DOSI, 2005; SAVIOTTI, 2001; SAVIOTTI; PYKA, 2004) .

En esa dirección, se presentan en forma estilizada las tres corrientes considerando los factores que determinan la innovación y el tipo de intervención que se podría derivar del marco teórico en el que están fundamentadas. Adicionalmente, el artículo intenta establecer algunos puentes de diálogo entre esas corrientes para 
identificar sus complementariedades, sus diferencias y los elementos teóricos que fundamentan las políticas de innovación.

El resto del artículo se estructura de la siguiente manera. En la segunda sección se presentan los aportes conceptuales de las tres corrientes. En la tercera sección se plantea la triangulación de esas corrientes para discutir la complementación de sus basamentos teóricos y sus recomendaciones de política. En la cuarta sección se parte de esta complementación y se incorporan al debate las especificidades que plantea el caso de Latinoamérica ${ }^{1}$ para re-pensar la política de innovación. Por último, en la quinta sección se presentan las principales conclusiones.

\section{Argumentos conceptuales sobre la intervención a partir de políticas de promoción de la innovación}

La intervención en política de innovación orientada a la generación de procesos de desarrollo se justifica desde diferentes enfoques, que acuerdan en la importancia de la intervención pero disienten en sus argumentos y formas.

Desde una perspectiva más cercana al mainstream, se generaron en forma reciente diversos esfuerzos que apuntan a incorporar elementos del enfoque de sistemas de innovación a los argumentos tradicionales centrados en fallas de mercado. En particular, la propuesta del BID (CRESPI et al., 2014, 2011) retoma tres ideas planteadas por este enfoque. En primer lugar, reconoce que la innovación es el resultado de múltiples esfuerzos complementarios que van más allá de los gastos en $\mathrm{I}+\mathrm{D}$ e incluyen la adquisición de maquinaria, la incorporación de conocimiento externo, la capacitación, la contratación de consultorías y las actividades de diseño e ingeniería. De esta manera, la presencia de fallas de mercado puede obedecer también a la sub-inversión de este tipo de esfuerzos y no únicamente a la ausencia de I+D. La segunda idea es que las posibilidades de innovar no dependen sólo del acceso al financiamiento sino también de la disponibilidad de recursos complementarios como la calidad y cantidad del capital humano y la infraestructura tecnológica. Consideran también que en el marco del nuevo paradigma tecnológico, las instituciones y mercados responsables del desarrollo de estos recursos muestran una tasa de respuesta muy lenta frente al ritmo de la demanda de conocimiento de los sectores productivos. En este contexto, se sostiene que la intervención de política debería orientarse no sólo a ofrecer financiamiento sino también a generar incentivos para

1 Si bien en este artículo nos referimos a América Latina en forma agregada, reconocemos la existencia de fuertes heterogeneidades entre los países que la conforman. El análisis planteado pretende abordar algunos hechos estilizados transversales a esos países. 
que las instituciones científicas y educativas respondan en forma más adecuada y con mayor rapidez a las demandas del sistema productivo. En tercer lugar, se reconoce la importancia del conocimiento tácito y se propone intervenir sobre los problemas de coordinación que se generan entre los actores involucrados en la generación y difusión de ese conocimiento.

En contraposición a estas ideas, los diferentes enfoques evolucionistas neo-schumpeterianos que discutiremos en el artículo han realizado importantes contribuciones a la agenda de debate sobre la política de innovación. Desde estas perspectivas, se aportan un conjunto de micro fundamentos que discuten la inconsistencia de la idea de intervención por fallas de mercado (DOSI, 1988, 2014; METCALFE, 1995). Entre ellos destacan que la racionalidad limitada, la incertidumbre radical y la micro-heterogeneidad dan lugar a diferencias en el acceso a la información, a la tecnología y a las externalidades que se generan en el entorno en el que compiten las firmas. Esto explica por qué las oportunidades endógenas de innovación no son percibidas de la misma forma por los actores, por qué los esfuerzos y resultados de innovación son altamente heterogéneos y por qué, en un mismo mercado, coexisten firmas con muy desiguales tasas de beneficios y niveles de productividad.

Partiendo de estas premisas epistemológicas, la literatura evolucionista neo-schumpeteriana considera que la dinámica de un sistema económico no converge a situaciones de óptimos paretianos. Por el contrario, la heterogeneidad y divergencia persistentes no resultan de la existencia de fallas de mercado sino de dinámicas evolutivas diferenciales, que tienen características path dependence y que funcionan fuera del equilibrio. ${ }^{2}$

A pesar de que la mayoría de las corrientes que discutiremos aceptan estos elementos epistemológicos, la literatura heterodoxa sobre economía de la innovación no constituye un corpus homogéneo (SUZIGAN; FURTADO, 2006). En este artículo y siguiendo la taxonomía propuesta por Robert y Yoguel (2016), identificamos tres corrientes de pensamiento evolucionista neo-shumpeteriano: sistemas de innovación, auto-organización, y causación acumulativa. Estas tres corrientes comparten una perspectiva epistemológica evolucionista basada en las siguientes premisas: realismo, final abierto y no reduccionismo (Robert, Yoguel y Lerena, 2017).

En el enfoque de sistemas de innovación, el realismo se manifiesta en el reconocimiento de las interacciones entre actores como canales específicos para la la literatura, Bleda y Del Rio (2013) identifican lo que se denomina "disfunciones" (LLERENA; MATT, 2005), "debilidades" (BERGEK et al., 2010) y "problemas sistémicos” (CHAMINADE; EDQUIST, 2006; CHAMINADE et al., 2009). 
circulación de información y conocimiento en contraposición a la idea de spillovers de conocimiento que no requieren acciones intencionales de los agentes. La dinámica de final abierto se asocia a la noción de divergencia entre sistemas, que no sólo es persistente sino que puede ampliarse por los impulsos que genera la dinámica de la innovación. El no reduccionismo está centrado en los feeedbacks positivos entre componentes y su influencia en los patrones agregados.

En la corriente de auto-organización el realismo se deriva de su concepción del proceso competitivo, que tiene lugar fuera de equilibrio y que resulta de las interacciones entre los elementos de diferentes poblaciones. El final abierto se manifiesta en cambios continuos del comportamiento de los actores en el proceso competitivo. El no reduccionismo alude a que cada elemento de la población constituye un sistema.

Finalmente, en la corriente de causación acumulativa el realismo se expresa en la co-evolución de fuentes Keynesianas, Schumpeterianas y Kaldorianas del crecimiento. El final abierto se refleja en la visión del crecimiento como proceso de transformación y no como un lugar de llegada a un estado estacionario (FAGERBERG; VERSPAGEN, 2002); lo que conduce a la existencia de patrones de divergencia persistente. Finalmente, el no reduccionismo se manifiesta en el carácter no lineal del mecanismo de causación acumulativa.

\subsection{El enfoque de sistemas de innovación}

En sus diversas vertientes, el enfoque de Sistemas de Innovación ${ }^{3}$ plantea que las capacidades y la articulación de sus componentes (empresas, universidades, centros tecnológicos, gobiernos, cámaras empresariales, consultores, etc.) dan lugar a la emergencia de sistemas con características idiosincrásicas (LUNDVALL et al., 2009). La innovación es el resultado emergente y no planeado de la interacción de sus componentes y de mecanismos de retroalimentación entre los elementos del sistema (EDQUIST, 1997; CHAMINADE et al., 2011; CASSIOLATO; LASTRES, 2008). En tal sentido, un sistema de innovación puede ser concebido como una herramienta analítica que se manifiesta en un amplio gradiente que va desde su virtual ausencia, derivada de articulaciones y capacidades débiles de los componentes, hasta un funcionamiento virtuoso que potencia la construcción de capacidades y

3 Incluye la clásica perspectiva de sistemas nacionales de innovación (FREEMAN, 1987; LUNDVALL, 1992; NELSON, 1993; EDQUIST, 1997), sistemas sectoriales (MALERBA; ORSENIGO, 1997; BRESCHI; MALERBA, 2000) y sistemas locales (ASHEIM; ISAKSEN, 1997; COOKE et al., 1997; BOSCHMA; MARTIN, 2010). 
la generación de ventajas competitivas dinámicas (EDQUIST; HOMMEN, 1999; LUNDVALL et al., 2011; CHAMINADE et al., 2011).

Las características estructurales de un sistema de innovación pueden constituir tanto elementos restrictivos como potenciadores del desarrollo. Por eso, la presencia de vinculaciones que estimulan procesos de aprendizaje e intercambio de conocimientos está condicionada por el nivel que alcanzan las capacidades de las organizaciones. En esa dirección, para que los componentes del sistema se puedan articular y apropiar de las externalidades se requiere un umbral mínimo de capacidades (YOGUEL; BOSCHERINI, 2001).

Desde esta visión, Edquist (1997) plantea que la noción de optimalidad que se deriva de comparar el sistema de innovación existente con su configuración ideal constituye un ejercicio imposible y de escaso interés. Por el contrario, interesa entender las diferencias y trayectorias divergentes de Sistemas de Innovación y no una comparación con un sistema ideal.

Las recomendaciones de política que se derivan de esta corriente se centran en identificar los elementos que bloquean el funcionamiento virtuoso del sistema y en proponer soluciones orientadas a mejorar las capacidades y conexiones de sus componentes. El objeto de la intervención refiere a dos componentes claves del proceso de aprendizaje: uno que enfatiza la importancia del conocimiento experimental y tácito -Doing, Using and Interacting (DUI)- y otro que se basa en la generación de conocimiento a partir de la relación universidad-empresa y de las actividades de I+D -Science, Technology and Innovation (STI)- (LUNDVALL, 2007). Siguiendo el planteo de Chaminade et al. (2011), la política pública de innovación debería combinar ambos tipos de aprendizaje, independientemente del estadio de desarrollo. La competitividad de las firmas depende de la combinación de estos dos aprendizajes a partir de una mejora de capacidades que considere la capacitación, la educación formal y los aprendizajes organizacionales.

Sin embargo, Lundvall (2007) señala que cuando interesa entender los problemas de los países en desarrollo el foco de la política debería centrarse en una secuencia que comience con la construcción de capacidades centradas en DUI y continúe con STI. ${ }^{4}$ El proceso de generación de competencias requiere entonces poder atravesar distintas etapas que incluyen el desarrollo de capacidades ingenieriles, de diseño, de gestión y finalmente de I+D formal e informal. Por eso, cuando

4 Es posible identificar cierta contradicción en la literatura de Sistemas de Innovación cuando aborda los problemas del desarrollo, en tanto no queda claro si los procesos de aprendizaje DUI y STI deben impulsarse en forma secuencial o simultánea en los países en desarrollo. 
el tipo DUI de aprendizaje no está suficientemente desarrollado, la función de las universidades no es generar procesos co-evolutivos de innovación con empresas sino fundamentalmente suministrar recursos humanos calificados que puedan mejorar las capacidades de los actores del sistema. Como se discutirá en la tercera sección, esta visión secuencial de la política de innovación para países de menor desarrollo relativo, puede conducir a situaciones de lock-in cuando las políticas DUI no se complementan con las de tipo STI. En esos casos, se puede generar una división internacional del trabajo que refuerce la brecha de productividad entre países en desarrollo y desarrollados.

Desde el punto de vista normativo, la política de innovación que se deriva de esta corriente se enfoca en la promoción del aprendizaje colectivo y la articulación de los actores mediante políticas de transferencia de conocimientos y tecnología (EDQUIST; HOMMEN, 1999; LUNDVALL, 1992). En ese sentido, se derivan recomendaciones de política principalmente de carácter horizontal y no selectivas, dado que no hay una preocupación teórica por el efecto de la estructura productiva en el desarrollo de los sistemas. ${ }^{5}$

\subsection{La literatura sobre auto-organización}

La literatura centrada en auto-organización ${ }^{6}$ (METCALFE, 1995; DOPFER; FOSTER; POTTS, 2004; POTTS, 2000) define la innovación como un aumento en la variedad de bienes, servicios y/o procesos, que la selección del mercado reduce vía la desaparición de firmas o vía una reducción de su cuota de mercado ${ }^{7}$. Esta visión "cíclica" de los procesos de innovación, de raíz schumpeteriana, se sustenta en la identificación de los mecanismos de variación, selección y retención, y en la existencia de feedbacks entre esos mecanismos en el proceso de competencia poblacional.

El cambio tecnológico es entendido como el resultado de dos fuerzas que coexisten y se retroalimentan mutuamente (ANDERSEN, 2004; CANTNER,

5 En la literatura de SI, la importancia de la estructura productiva es abordada por el enfoque de sistemas sectoriales de innovación (MALERBA; ORSENIGO, 1997), que destaca la existencia de regímenes tecnológicos con diferentes condiciones de acumulatividad, apropiabilidad, oportunidad y conocimiento de base.

6 En esta corriente, la población alude a un espacio específico en el que compiten las firmas.

7 Desde la corriente de auto-organización se puede reinterpretar la idea de sistema de innovación considerándolo como un conjunto de poblaciones en las que las firmas compiten y tienen vinculaciones tanto con organizaciones de su población como de otras. El concepto de sistema no se puede escindir del proceso de competencia, de los valores de las características de la selección poblacional y de las instituciones del mercado que condicionan el proceso de selección. Esta es una diferencia significativa de los autores de esta corriente con la de Sistemas de Innovación. 
2007): la generación de variedad impulsada por la introducción de novedad en la población; y el proceso de selección del mercado, responsable de que firmas con diferentes valores en las características de selección poblacional -productividad, costos unitarios de trabajo, entre otros- tengan diferentes tasas de crecimiento y, por lo tanto, distintas cuotas de mercado.

Una consecuencia del proceso de selección es que la media de la característica poblacional -por ejemplo, la productividad-va cambiando a lo largo del tiempo. Si el proceso de selección funciona adecuadamente, las firmas con niveles de productividad inferiores al promedio o bien disminuyen su participación en la población o son expulsadas del mercado, lo que aumenta en consecuencia la productividad media de la población. Metcalfe (2010) modeliza esta dinámica utilizando el mecanismo de replicator dynamics, según el cual la cuota de mercado de una firma evoluciona de acuerdo a la distancia entre su nivel de productividad y la productividad media de la población bajo estudio. Así, un sistema necesita generar variedad a partir de la innovación, y poner en marcha mecanismos de selección que modifiquen las características de la población. Dado que la selección reduce la variedad de firmas, la innovación es clave para generar novedad y variedad en la población (METCALFE, 1995; DOPFER, 2001, 2005; FOSTER, 2005; DOPFER; FOSTER; POTTS, 2004; POTTS, 2000; WITT, 1997; CANTNER, 2007; POTTS, 2000). ${ }^{8}$

Las recomendaciones de política de innovación que surgen del enfoque de auto-organización son de carácter implícito. Se centran en la necesidad de considerar la co-evolución entre los procesos de competencia, la generación de procesos de innovación y la dinámica evolutiva (METCALFE; FOSTER; RAMLOGAN, 2006). En esta línea, los principales objetivos de política de innovación se orientan al diseño e implementación de instrumentos que contribuyan a regular y a fomentar el proceso de competencia de las firmas en el mercado (variación, selección y retención), a partir de la creación de instituciones, normas y estándares y al desarrollo de las capacidades tecnológicas de las firmas. Se plantea además una intervención experimental en el plano micro y meso-económico y con carácter transversal a los sectores económicos que se sustenta en el desarrollo de capacidades y en el diseño de instituciones de mercado que eviten las fallas de selección, que son las únicas que reconoce este enfoque.

8 Desde esta perspectiva el mercado es concebido como una construcción social históricamente contingente. Es decir, como una propiedad que emerge de los comportamientos heterogéneos de firmas que compiten en desequilibrio y que están condicionados por las instituciones, normas, rutinas y estándares construidos (KIRMAN, 2010). 


\subsection{La literatura sobre causación acumulativa}

Los aportes de los autores del enfoque de causación acumulativa (DOSI, 2000; MARENGO; DOSI, 2005; DOSI; LEVINTHAL; MARENGO, 2003; SAVIOTTI, 2001; SAVIOTTI; PYKA, 2004; DOSI, 2014; SAVIOTTI; PYKA, 2002; LEE, 2013) complementan las perspectivas anteriores, siguiendo las ideas de Kaldor sobre la existencia de mecanismos de causación acumulativa y su efecto sobre la dinámica de retornos crecientes, externalidades de red, crecimiento de la demanda y productividad.

Los cambios en la composición del sistema económico derivados de la demanda y la emergencia de innovaciones co-evolucionan (SAVIOTTI; PYKA, 2002) a partir del siguiente mecanismo: la saturación de la demanda en ciertos sectores induce cambios en factores de oferta, como la tecnología, que generan variedad. Esta variedad puede ser de dos tipos: relacionada con la estructura productiva inicial, que refleja los procesos de diferenciación dentro de un mismo sector; y no relacionada, ${ }^{9}$ que da lugar al desarrollo de nuevos sectores.

Ambos tipos de variedad son dimensiones complementarias y necesarias de un proceso de desarrollo económico que conduzca a disminuir la brecha entre sistemas productivos. La centralidad del enfoque está puesta en una dinámica sistémica de co-evolución entre los procesos de destrucción creativa -asociados a la generación de variedad- y la dinámica de la demanda que, en los casos positivos, da lugar a que la oferta sea absorbida. Como plantea Saviotti (2001), el crecimiento continuo de la productividad y la saturación de la demanda crean un cuello de botella para el desarrollo, que puede ser compensado por la emergencia de nuevos sectores.

En este marco, la estructura productiva tiene un rol preponderante en la dinámica económica dado que, por un lado, la generación de variedad depende de la creación de nuevos sectores y de las posibilidades de diferenciación en los sectores existentes y, por otro, el fenómeno de divergencia entre sistemas económicos de distinto grado de desarrollo relativo es en parte explicado por la composición de la estructura.

9 Esta última requiere un significativo aumento de la creatividad en el sistema y el aprovechamiento de oportunidades y esfuerzos de innovación preferentemente no incorporados y de tipo radical. 
Este proceso de divergencia es resaltado también por dos corrientes de pensamiento emparentadas con el grupo de causación acumulativa: la perspectiva neo-estructuralista (PERES, 2006; CIMOLI et al., 2009) y la post-keynesiana (SETTERFIELD, 2010; O’HARA, 2008; CHICK; DOW, 1998). En ambos casos, el fenómeno de divergencia es explicado por las diferencias en los niveles de capacidades necesarias para absorber las tecnologías y diseños desarrollados en los países de frontera y por la composición de la estructura productiva en los países en desarrollo.

Las recomendaciones de política incluyen una manifiesta preocupación por disminuir la brecha de productividad que los países en desarrollo tienen con las economías de ingresos altos. Por lo tanto, todo proceso de desarrollo requiere que las estructuras productivas se direccionen hacia dinámicas que generen innovación y que den lugar a feedback positivos y rendimientos crecientes de tipo dinámico. Por eso, la generación de oportunidades de aprendizaje no es independiente de la especialización sectorial y de la dinámica de la demanda. Así, se deduce que la intervención debe tener por objetivo central la generación de procesos de cambio estructural virtuosos caracterizados por un aumento significativo del peso de sectores que combinan eficiencia schumpeteriana (sectores intensivos en conocimiento y con rendimientos crecientes) y eficiencia keynesiana (sectores de rápido crecimiento de la demanda interna y externa).

\section{La articulación de las tres perspectivas evolucionistas neo-schumpeterianas}

Las corrientes teóricas presentadas colocan el énfasis en dimensiones diferentes y complementarias que pueden ser articuladas en un mismo enfoque con el objetivo de enriquecer el análisis tanto sobre el rol de la innovación en el desarrollo como sobre los argumentos que justifican la intervención.

En lo Cuadro 1 se presenta en forma estilizada los elementos de cada enfoque que permiten efectuar una triangulación de los mismos, en torno a las características de la innovación, los objetivos de política y los niveles de intervención que se derivan de cada corriente. 
CUADRO 1

Síntesis de las tres corrientes evolucionistas neo-schumpeterianas

\begin{tabular}{|c|c|c|c|}
\hline Corriente & $\begin{array}{l}\text { Características de la } \\
\text { innovación }\end{array}$ & Objetivos de política & $\begin{array}{c}\text { Niveles de } \\
\text { intervención }\end{array}$ \\
\hline $\begin{array}{l}\text { Sistemas de } \\
\text { innovación }\end{array}$ & $\begin{array}{l}\text { - Innovación como } \\
\text { proceso sistémico y } \\
\text { emergente. } \\
\text { - Divergencia de } \\
\text { sistemas. }\end{array}$ & $\begin{array}{l}\text { - Fomentar los procesos de } \\
\text { aprendizaje de tipo DUI y STI } \\
\text { en los componentes del sistema } \\
\text { (construcción de capacidades). } \\
\text { - Favorecer la conexión de los } \\
\text { componentes del sistema. }\end{array}$ & Micro / Meso \\
\hline $\begin{array}{l}\text { Auto- } \\
\text { organización }\end{array}$ & $\begin{array}{l}\text { - Co-evolución entre la } \\
\text { generación de variedad } \\
\text { y los mecanismos de } \\
\text { selección en el proceso } \\
\text { de competencia }\end{array}$ & $\begin{array}{l}\text { - Aumentar las capacidades para ge- } \\
\text { nerar innovaciones que aumenten la } \\
\text { variedad } \\
\text { - Regular el proceso de competencia } \\
\text { de las firmas en el mercado } \\
\text { - Fortalecer las instituciones de } \\
\text { mercado de modo de poder operar } \\
\text { sobre los mecanismos de selección. }\end{array}$ & Micro/Meso \\
\hline $\begin{array}{l}\text { Causación } \\
\text { acumulativa y } \\
\text { divergencia }\end{array}$ & $\begin{array}{l}\text { - Innovación como } \\
\text { emergente del } \\
\text { proceso de causación } \\
\text { acumulativa. } \\
\text { - Innovación como } \\
\text { generación de variedad } \\
\text { relacionada y no } \\
\text { relacionada. }\end{array}$ & $\begin{array}{l}\text { - Cambio estructural. } \\
\text { - Aumento de la eficiencia keynesiana } \\
\text { y schumpeteriana. }\end{array}$ & Meso/Macro \\
\hline
\end{tabular}

Fonte: Elaboración propia.

La propuesta de articulación de las tres perspectivas se sustenta, en primer lugar, en las ventajas de contar con un enfoque analítico que combine las dimensiones micro, meso y macro, enfatizadas de distinta forma por cada una de las corriente consideradas. Mientras que la perspectiva de auto-organización se centra en un plano micro y meso, la de Sistemas de Innovación enfatiza el plano mesoeconómico y la de causación acumulativa aborda los niveles meso y macro. De esta manera, siguiendo a Dopfer, Foster y Potts (2006), el plano meso podría ser una dimensión útil para la articulación de las corrientes. Así, la integración y triangulación de los enfoques busca generar un marco analítico que permita entender la emergencia de los procesos de innovación, donde confluyen las dimensiones micro (capacidades), 
meso (proceso de competencia, patrón de especialización y vinculaciones) y macro (procesos agregados de causación acumulativa con un rol clave de la interacción entre oferta y demanda).

En segundo lugar, la necesidad de una triangulación surge de la identificación de denominadores comunes que actúan como ejes articuladores en cada enfoque y de elementos ausentes en unos y presentes en otros cuyo potencial explicativo puede aumentar cuando son analizados en forma conjunta.

El denominador común, que es transversal y a la vez clave en los tres enfoques, es el rol de las capacidades, ya sea de las firmas, de otros componentes del sistema y de las instituciones para explicar la emergencia de procesos de innovación.

Los elementos complementarios, que están presentes en alguno de los enfoques pero no en los tres a la vez, son: el perfil de especialización sectorial; el rol del proceso de competencia; y el carácter experimental de la política.

En relación al perfil de especialización, ni el enfoque de Sistemas de Innovación ni el de auto-organización lo incluyen como un eje central en la discusión teórica y normativa. En la primera corriente, los componentes centrales del sistema son las organizaciones y sus vínculos. Desde esa perspectiva la intervención se justifica a partir de la existencia de fallas sistémicas y las recomendaciones de política aluden a crear las instituciones adecuadas al margen del perfil de especialización. Al no considerar la estructura productiva no se tiene en cuenta que, por ejemplo, los vínculos user-producer y universidad-industria asumen distintas formas y características en cada sector según el tipo e intensidad del conocimiento involucrado. En la segunda corriente (auto-organización), el patrón de especialización constituye una propiedad emergente sobre la que el enfoque no plantea actuar. ${ }^{10}$ Por lo tanto, ninguno de estos dos enfoques discute qué sectores promover. Esta discusión aparece con más claridad en el enfoque de causación acumulativa que plantea que los procesos de desarrollo y el fenómeno de divergencia entre países no es independiente de la estructura productiva. Esta dimensión, como ámbito de actuación, es central en esta corriente porque considera que la innovación está condicionada por la estructura productiva inicial y porque el peso relativo de los diversos sectores en la economía depende en parte de las posibilidades de innovar. Esto se manifiesta en los efectos de retroalimentación entre estructura productiva, demanda e innovación. A diferencia del enfoque de Sistemas de Innovación, la divergencia persistente entre los senderos

10 Esta corriente plantea explícitamente la idea de cambio estructural pero con un significado distinto al considerado por la corriente de CA, aludiendo sólo a modificaciones del peso de las empresas en la población en la que compiten. 
de desarrollo se explica por la presencia de fenómenos de lock-in que bloquean la dinámica del cambio estructural y limitan las posibilidades de generar procesos de especialización que combinen eficiencia schumpeteriana y eficiencia keynesiana. Es desde la perspectiva meso que se puede interpelar a las corrientes de Sistemas de Innovación y auto-organización en relación a la especialización productiva.

Cuando se incorpora al análisis de política el debate sobre cambio estructural, el enfoque de Sistemas de Innovación puede contribuir a la discusión a partir de la identificación de las instituciones y de los mecanismos DUI y STI de generación del conocimiento necesario, adaptados a sectores específicos. A su vez, desde el enfoque de auto-organización el análisis del proceso de competencia aporta elementos micro y mesoeconómicos que permiten discutir sobre la factibilidad de desarrollar nuevos sectores y actuar sobre el cambio de perfil de especialización. En particular, aluden a las instituciones de mercado necesarias (normas, estándares, regulaciones), que posiblemente difieren entre un sector y otro y a las formas predominantes de mercado en términos de salida, entrada y formas cómo funciona el proceso de competencia.

El análisis del proceso de competencia y su influencia en la generación de capacidades e innovaciones constituye una dimensión casi ausente en la literatura de Sistemas de Innovación y causación acumulativa. Por el contrario, adquiere centralidad en el enfoque de auto-organización. Este enfoque plantea la necesidad de actuar sobre la dinámica co-evolutiva entre los procesos de competencia, generación de capacidades e innovación, con consecuencias sobre el aumento/disminución de la participación de las firmas que compiten en una determinada población.

La introducción del proceso de competencia en un análisis que combine los tres enfoques requiere incorporar los factores que actúan sobre los mecanismos de selección y la forma como se combinan con el proceso de desarrollo de capacidades. También requiere estudiar de qué manera actúan sobre el proceso de competencia los componentes, conexiones y feedbacks de los sistemas de innovación.

A la vez, la articulación entre la demanda (como elemento diferenciador del enfoque de causación acumulativa) y el proceso de competencia introduciría en los mecanismos de replicador dinámico (METCALFE, 2010) una variable adicional que no es planteada en la corriente de auto-organización. Se podría pensar que los procesos de selección, variedad y retención serían influidos de distinta forma en poblaciones con diferentes elasticidades ingreso de la demanda. Por ejemplo, cuando la elasticidad ingreso de la demanda es elevada, los mecanismos de eficiencia keynesiana co-evolucionan con mecanismos de eficiencia schumpeteriana (HOLLAND; PORCILE, 2005) y, por lo tanto, la innovación constituye la característica de selec- 
ción poblacional. En cambio, esto no ocurriría en poblaciones con baja elasticidad ingreso de la demanda.

Finalmente, el diseño e implementación de políticas de innovación incluye, sobre todo en los enfoques de Sistemas de Innovación y auto-organización, un fuerte componente experimental que responde a la concepción de una realidad dinámica y de final abierto, con agentes que tienen racionalidad acotada, información imperfecta e incertidumbre radical. La integración del carácter experimental a la triangulación propuesta se corresponde con la visión que las tres corrientes comparten sobre sistemas de ideas abiertos, interesados en analizar "el comportamiento de los agentes y las estructuras económicas y sociales y su interrelación en un contexto histórico y evolutivo" (PEREZ CALDENTEY, 2015, p52).

\section{Las especificidades de América Latina en la articulación de las corrientes evolucionistas neo-schumpeterianas}

El análisis de los procesos de innovación en América Latina desde la articulación planteada de los tres enfoques teóricos revisados, requiere ser aggiornado teniendo en cuenta un conjunto de hechos estilizados de la dinámica productiva y de innovación resaltados por la literatura de la región (AROCENA; SUTZ, 2006; ERBER, 1983; BIANCHI et al., 2008; BENAVENTE; CONTRERAS, 2008; KUPFER; AVELLAR, 2008; ARZA; LÓPEZ, 2008; ERBES et al., 2010). Estos hechos estilizados se manifiestan en: actividades de innovación con reducido peso de la inversión en $\mathrm{I}+\mathrm{D} ;^{11}$ fuerte sesgo del sector público como fuente del gasto; reducida capacidad de absorción de las firmas que limita las vinculaciones con otros agentes del sistema; prevalencia de actividades informales de innovación; y limitado rol de las regulaciones e instituciones del mercado.

En este marco, consideramos que una adaptación de los enfoques revisados permitiría comprender la dinámica productiva y de innovación a partir de sus elementos centrales. Estos elementos son el rol de las capacidades, la centralidad del proceso de competencia y la co-evolución entre estructura productiva y demanda.

11 En Argentina, sólo el 8\% de las firmas manufactureras declaró contar con un laboratorio de I+D en 2012, lo que contrasta con el 60\% que declaró haber realizado algún esfuerzo de innovación (MINCyT, 2015). En Brasil, mientras que 28\% de las firmas manufactureras realizó alguna actividad de innovación en 2011, sólo 3,7\% realizó actividades de I+D de forma continua (PINTEC, 2016). En Chile, sólo el 1,6\% de las empresas declaró contar con un laboratorio de I+D en 2012, versus el 27\% que declaró haber innovado (EIE, 2014). En Uruguay, 7\% de las empresas manufactureras y de servicios declararon realizar actividades de I+D entre 2010 y 2012, al tiempo que más del 20\% realizó esfuerzos de innovación (ANII, 2015). En México, mientras que $6,1 \%$ de las empresas manufactureras contaba con laboratorios de I+D en 2012, alrededor del $15 \%$ declaró haber introducido nuevos productos o procesos (ESIDET-MBN, 2012). 
En primer lugar, partiendo del enfoque de Sistemas de Innovación, Arocena y Sutz (2006, p. 2) sostienen que el carácter sistémico de la innovación no se puede dar por supuesto; "se realiza, por cierto, a través de vínculos e interacciones entre actores diversos, pero unos y otras suelen ser frágiles, episódicos y escasos. Los Sistemas de Innovación son más potenciales que reales. Esto tiene importancia teórica, pero sobre todo práctica: las políticas para la innovación en el Subdesarrollo no pueden dar por sentado que los 'sistemas' existen y funcionan como tales". Las posibilidades de desarrollar capacidades son menores por la escasa presencia de espacios interactivos que reduce considerablemente las oportunidades de "aprender haciendo", "aprender resolviendo" y "aprender interactuando".

Por su parte, en el marco de una región con países que, en su mayoría, tienen un perfil de especialización de bajo contenido tecnológico y de conocimiento, pierde sentido el excesivo énfasis puesto en las actividades de I+D formal como objetivo de las política orientadas a disminuir la brecha de productividad. El rol dado a la intensidad de la $\mathrm{I}+\mathrm{D}$ como indicador central de los esfuerzos de innovación de las firmas excluye gran parte de las actividades de innovación que no se asocian a la generación endógena de conocimiento (Barletta et al, 2016). En cambio, se les asigna escasa relevancia a los indicadores asociados a los procesos de aprendizaje DUI como la creación de capacidades y rutinas ingenieriles, de diseño y de gestión, que son claves para alcanzar un umbral mínimo de capacidades para encarar esfuerzos de innovación más sofisticados. Desde esta perspectiva de Sistemas de Innovación aggiornada a América Latina, las innovaciones no-tecnológicas y a las características de los procesos de aprendizaje necesarios para producir, difundir y utilizar conocimientos tecnológicos adquieren un papel relevante (JOHNSON et al., 2003; LUNDVALL et al., 2011; CHAMINADE et al., 2011). Como plantean estos autores los sistemas de innovación de países en desarrollo son emergentes y se caracterizan por la débil presencia de los building blocks constitutivos del sistema y por los reducidos vínculos entre los componentes. En ese contexto, la ausencia de organizaciones intermedias limita los procesos de creación de competencias y la innovación.

Las políticas de vinculación pueden no ser efectivas cuando el nivel de capacidades es bajo y la micro-heterogeneidad se manifiesta en una elevada distancia cognitiva y tecnológica entre los componentes del sistema que limita los potenciales beneficios de la interacción. Sin embargo, una orientación de política sesgada hacia procesos de aprendizaje de formas DUI más que STI podría generar una división del trabajo que refuerce la brecha de productividad inicial entre América Latina y los países desarrollados. 
En segundo lugar, la reinterpretación del enfoque de auto-organización para que sea útil en América Latina requiere considerar que los procesos de selección en la región no sólo se dan necesariamente sobre la base de las características poblacionales que identifica esta perspectiva. En ciertos casos, la selección no depende de la productividad de las firmas y/o de la existencia de estándares de calidad sino fundamentalmente de factores precio de la competitividad, que se explican por una composición de la estructura productiva con predominio de sectores con alta eficiencia factorial y baja eficiencia schumpeteriana y keynesiana.

En tercer lugar, una propuesta evolucionista neo-schumpeteriana de política de innovación para América Latina desde la perspectiva de causación acumulativa resalta la importancia de generar un cambio estructural que conduzca a la disminución de la brecha de productividad, al aumento del empleo de calidad y a una distribución del ingreso más equitativa. Sin embargo, este proceso no puede entenderse desde un punto de vista reduccionista que considere únicamente la creación de sectores de alta productividad en la matriz productiva. Las características estilizadas sobre la dinámica productiva y de innovación de los países de América Latina requieren diseñar políticas de innovación orientadas a "combinar la acumulación de conocimiento y la diversificación de la estructura productiva. La primera genera las posibilidades; la segunda las concreta" (PERES, 2006, p. 72). Dado que no cualquier estructura permite alcanzar altos niveles de competitividad, el perfil de especialización del que parten los países de la región es un limitante para generar procesos de cambio estructural. Las sugerencias de Saviotti y Pyka (2004), desde el enfoque de causación acumulativa, de generar variedad relacionada y no relacionada en el corto y largo plazo, respectivamente, no son igualmente aplicables a cualquier economía. Las posibilidades de diferenciar la oferta productiva son mucho más bajas en países donde predomina la producción de commodities con un alto nivel de estandarización y un reducido espacio para innovar (BARLETTA et al., 2012).

En relación a estas cuestiones, la literatura reciente se bifurca cuando se debate sobre las potencialidades y límites de una especialización productiva centrada en recursos naturales (PAMPLONA; CACCIAMALI, 2017). Por un lado, diversas posiciones, entre las que resaltan Pérez (2010 y 2012), Bielschovsky (2012), Furtado (2008), Ferranti et al. (2002) y Frankel (2012) enfatizan que la abundancia de recursos naturales en la región se puede convertir en una ventaja competitiva por la hiper-segmentación y des-commoditización de los mercados de productos intensivos en recursos naturales desencadenados por el paradigma tecno-económico vigente. Otras posturas, como la de Cimoli, Dosi y Stiglitz (2009) y Cypher (2014) 
tienen una visión contraria enfatizando que una especialización centrada en recursos naturales es intensiva en capital, demanda poca mano de obra calificada y alcanza un crecimiento menor en el largo plazo. En la misma dirección, Barletta et al (2012) ponen en duda que una especialización en nichos de productos intensivos en recursos naturales puedan generar procesos de cambio estructural, en especial por la reducida eficiencia keynesiana y schumpeteriana que caracterizan ese tipo de productos. $^{12}$

La idea de cambio estructural que está por detrás de ese debate alude a un proceso de transformación cualitativa y cuantitativa que se refleja en: un aumento generalizado de las capacidades y eficiencia de las firmas e instituciones; el creciente peso de las interconexiones de los componentes del sistema que permite una mayor circulación del conocimiento y de la información; la generación de variedad relacionada y no relacionada; y la presencia de feedbacks positivos entre los componentes y los distintos niveles del sistema -micro, meso y macro- que den lugar a un aumento de las capacidades y a la aparición de rendimientos crecientes de tipo dinámico (ERBES et al., 2010). Así, una intervención que tenga esa perspectiva requiere desarrollar un proceso que actúe sobre las esferas micro, meso y macro, de modo de reducir los bloqueos existentes a la emergencia de los fenómenos de eficiencia schumpeteriana y keynesiana.

Las limitadas capacidades tecnológicas de los países de América Latina, y la elevada heterogeneidad existente entre los países miembros constituyen uno de los principales bloqueos en el sendero de crecimiento, que restringe las posibilidades de cambio estructural. Según Lee (2013), la problemática no radica ni en las fallas de mercado que propone el mainstream, ni en las fallas sistémicas de coordinación planteado por el grupo de auto-organización sino en las limitadas capacidades para generar diseño y marca propia en alta tecnología. La ausencia de estas capacidades promueve una tendencia hacia la adquisición de $\mathrm{I}+\mathrm{D}$ incorporada en bienes de capital (importados), la obtención de facilidades de producción vía licencias o patentes y la especialización en segmentos de ensamble con poco contenido tecnológico en cadenas globales de producción.

12 No es ajena a esta polémica la volatilidad de los precios de las commodities, acentuada desde mediados de la década del 2000. Sobre la base de información del FMI, el índice de precios de las commodities primarias, con base 2005=100, y tomando el mes de mayo de cada año, tuvo una tendencia creciente pero volátil: de un nivel de 130 en 2007, se duplica en 2008 llegando a 204, cae a 115 en 2009, vuelve a subir a 200 en 2011, desciende a 125 en 2015 y a 111 en 2017. 


\section{Conclusiones}

En este artículo hemos presentado en forma estilizada las ideas centrales sobre innovación e implicancias de política de tres corrientes evolucionistas neo-schumpeterianas: las centradas en sistemas de innovación, en auto-organización y en causación acumulativa. A partir de ellas, se ha planteado una articulación que retoma sus ideas predominantes y combina los elementos micro, meso y macro de cada perspectiva.

En el artículo hemos planteado que una ontología evolucionista neo-schumpeteriana no es compatible con un diseño de política centrada en fallas de mercado, las que no reflejan la forma como se manifiestan las dinámicas productivas en economías capitalistas, lejos del equilibrio, con fuerte heterogeneidad en el desempeño de las empresas e instituciones y con incertidumbres no modelables.

Los hechos estilizados de la dinámica innovadora de América Latina ponen de manifiesto que la búsqueda de procesos de cambio estructural requiere actuar simultáneamente en la mejora significativa de capacidades y articulaciones en los planos micro, meso y macroeconómicos que se derivan de la complementación de las tres corrientes analizadas. Desde la perspectiva micro el énfasis se centra en la promoción del desarrollo de capacidades -tecnológicas y no tecnológicas. Este tipo de intervención requeriría reflexionar críticamente sobre la centralidad qua asumen los instrumentos basados en la provisión de financiamiento para I+D cuando el contexto está caracterizado por fuertes restricciones previas en las capacidades de los actores que limitan el desarrollo de innovaciones.

Siguiendo la triangulación propuesta, estas intervenciones micro se complementan con otras de tipo meso y macro que promuevan cambios en el perfil de especialización y que faciliten los procesos de cambio estructural. Esto requiere repensar las modalidades de intervención pública de modo de priorizar programas que promuevan la generación de variedad relacionada y no relacionada y que tengan un carácter experimental. En esa dirección, aparece el interrogante acerca de cuáles son las condiciones para la emergencia de actividades caracterizadas por rendimientos crecientes, elevada eficiencia keynesiana y schumpeteriana, fuertes vinculaciones con el sistema de ciencia y tecnología, y una integración en los eslabones de mayor intensidad tecnológica de las cadenas globales de valor. 


\section{Referencias}

ANDERSEN, E. S. Population thinking, price's equation and the analysis of economic evolution. Evolutionary and Institutional Economics Review, v. 1, n. 1, p. 127-148, 2004.

AROCENA, R.; SUTZ, J. El estudio de la innovación desde el sur y las perspectivas de un nuevo desarrollo. CTS + I: Revista Iberoamericana de Ciencia, Tecnología, Sociedad e Innovación, n. 7, Septiembre-Diciembre 2006.

BIELSCHOWSKY, R. Estratégia de desenvolvimento e as três fontes de expansão no Brasil: um desenho conceitual. Economia e Sociedade, Campinas, v. 21, n. esp., p. 729- 47, dez. 2012. BARLETTA, F. ; ROBERT, V.; YOGUEL, G. Algunos comentarios sobre el artículo "Dinamismo tecnológico e inclusión social mediante una estrategia basada en los recursos naturales”, de Carlota Pérez. Revista Econômica, v. 14, n. 2, p. 55-61, 2012.

BERGEK, A.; JACOBSSON, S.; HEKKERT, M.; SMITH, K. Functionality of innovation systems as a rationale for, and guide to innovation policy. In: SMITS, R.; KUHLMANN, S.; SHAPIRA, P. (Ed.). Innovation policy, theory and practice. An international handbook. Elgar Publishers, 2010.

BLEDA, M.; DEL RÍO, P. The market failure and the systemic failure rationales in technological innovation systems. Research Policy, n. 42, p. 1039-1052, 2013.

BOSCHMA, R.; MARTIN, R. The handbook of evolutionary economic geography. Cheltenham, UK: Edward Elgar Publishing, 2010.

BRESCHI, S.; MALERBA, F. Sectorial Innovation Systems: technological regimes, schumpeterians dynamics and spatial boundaries. In: EDQUIST, C. (Ed.). Systems of innovation: technologies, institutions and organizations. London: Pinter Publishers/Cassell Academic, 1997. BREZNITZ, D.; ORNSTON, D. Scaling up and sustaining experimental innovation policies with limited resources: peripheral Schumpeterian development agencies. In: DUTZ, M. A. et al. (Ed.). Making innovation policy work. Learning from experimentation. OECD, World Bank, 2014. p. 247-284.

CASSIOLATO, J.; LASTRES, H. Discussing innovation and development: converging points between the Latin American school and the Innovation Systems perspective? Globelics, 2008 (Working Paper Series, n. 08-02).

CEPAL - Comissão Econômica para a América Latina. Cambio estructural para la igualdad. Una visión integrada del desarrollo. Santiago de Chile: Cepal, Julio 2012.

CHICK, V.; DOW, S. A post-keynesian perspective on the relation between banking and regional development. In: ARESTIS, P. (Ed.). Post-keynesian monetary economics: new approaches to financial modelling. Cheltenham, UK: Edward Elgar Publishing, 1998. p. 219-250. 
CIMOLI, M.; DOSI, G.; STIGLITZ, J. Industrial policy and development: the political economy of capabilities accumulation. Oxforf: Oxford University Press, 2009.

CIMOLI, M.; PORCILE, G.; ROVIRA, S. Structural change and the BOP-constraint: why did Latin America fail to converge? Cambridge Journal of Economics, v. 34, n. 2, p. 389-411, 2010.

CHAMINADE, C.; EDQUIST, C. From theory to practice. The use of the systems of innovation approach in innovation policy. In: HAGE, J.; DE MEEUS, M. (Ed.). Innovation, science and institutional change. Oxford: Oxford University Press, 2006.

CHAMINADE, C.; LUNDVALL, B.Å.; VANG, J.; JOSEPH, K. J. Designing innovation policies for development: towards a systematic experimentation-based approach, In: LUNDVALL, B. A.; JOSEPH, K. J.; CHAMINADE, C.; VANG, J. (Ed.). Handbook of innovation systems and developing countries: building domestic capabilities in a global setting. Cheltenham, UK: Edward Elgar Publishing, 2011.

COOKE, P.; URANGA, M.G.; ETXEBARRIA, G. Regional innovation systems: Institutional and organisational dimensions. Research Policy, v. 26, n. 4-5, p. 475-491, 1997.

CRESPI, G.; FERNANDEZ-ARIAS, E.; STEIN, E. Rethinking productive development: sound policies and institutions for economic transformation. Palgrave Macmillan, 2014.

CRESPI, G.; MAFFIOLI, A.; MOHNEN, P.; VÁZQUEZ, G. Evaluating the impact of science, technology and innovation programs: a methodological toolkit. Inter-American Development Bank, 2011.

CYPHER, J. Neoextraccionismo y primarización: términos de intercambio em América del Sur. In: GIRÓN, A. (Coord.). Democracia, financiarización y neoextraccionismo ante los desafios de la industrialización y el mercado de trabajo. México: Universidad Nacional Autónoma de México, Instituto de Investigaciones Económicas, 2014. p. 117-41.

DOPFER, K.; FOSTER, J.; POTTS, J. Micro-meso-macro. Journal of Evolutionary Economics, v. 14, n. 3, p. 263-279, 2004.

DOSI, G. Sources, procedures and microeconomic effects of innovation. Journal of Economic Literature, v. 26, n. 3, p. 1120-1171, 1988.

Dinámica y coordinación económica. Algunos elementos para un paradigma alternativo “evolucionista”. In: BARLETTA, F.; ROBERT, V.; YOGUEL, G. (Ed.). Tópicos de la teoría evolucionista neoschumpeteriana de la innovación y el cambio tecnológico Miño y Dávila: Universidad Nacional de General Sarmiento, 2014. v. 1.

DOSI, G.; FREEMAN, C.; NELSON, R.; SOETE, L. Technical change and economic theory. London: Pinter Publishers, 1989. 
DUTRENIT, G.; KATZ, J. Innovation, growth and development in Latin-America: Stylized facts and a policy agenda. Innovation: Management, Policy \& Practice, v. 7, n. 2-3, p. 105-130, 2005.

DUTZ, M. A.; KUZNETSOV, Y.; LASAGABASTER, E.; PILAT, D. Making innovation policy work: learning from experimentation. Paris: OECD Publishing, 2014.

EDQUIST, C. Systems of innovation: technologies, institutions, and organizations. Psychology Press, 1997.

EDQUIST, C.; HOMMEN, L. Systems of innovation: theory and policy for the demand side. Technology in Society, n. 21, p. 63-79, 1999.

ERBER, F. Technological dependence and learning revisited. In: MONTEIRO FILHA, D.; DELORME PRADO, L.; LASTRES, H. (Ed.). Estratégias de desenvolvimento, politica industrial e innovação: ensaios em memória de Fabio Erber. 2014. Brasília: BNDES, 1983.

ERBES, A.; ROBERT, V.; YOGUEL, G. Capacities, innovation and feedbacks in production networks in Argentina. Economics of Innovation and New Technology, v. 19, n. 8, p. 719-741, 2010.

FAGERBERG, J.; VERSPAGEN, B. Technology-gaps, innovation-diffusion and transformation: an evolutionary interpretation. Research Policy, v. 31, n. 8, p. 1291-1304, 2002.

FERRANTI, D. et al. From natural resources to the knowledge economy. Washington, D.C.: The World Bank, 2002 (World Bank Latin American and Caribbean Studies).

FOSTER, J. From simplistic to complex systems in economics. Cambridge Journal of Economics, v. 29, n. 6, p. 873-892, 2005.

FREEMAN, C. Technology policy and economic performance: lessons from Japan. London: Pinter Publishers, 1987.

FRANKEL, J. A. The natural resource curse: a survey of diagnoses and some prescriptions. Cambridge, MA: John F. Kennedy School of Government, Harvard University, 2012.

FURTADO, J. Muito além da especialização regressiva e da doença holandesa: oportunidades para o desenvolvimento brasileiro. Novos Estudos Cebrap, n. 81, jul. 2008.

HIRSCHMAN, A. O. The strategy of economic development. Clinton, MA: Yale University Press, 1958.

PORCILE, G.; HOLLAND, M. Brecha tecnológica y crecimiento en América Latina. In: CIMOLI, M. (Ed.). Heterogeneidad estructural, asimetrías tecnológicas y crecimiento en América Latina-LC/W. BID-Cepal,2005. p. 40-71.

JOHNSON, B.; EDQUIST, C.; LUNDVALL, B. Å. Economic development and the national system of innovation approach. In: FIRST GLOBELICS CONFERENCE. Rio de Janeiro, 2003. 
KIRMAN, A. Complex economics: individual and collective rationality. Routledge, 2010.

KNUDSEN, T. General selection theory and economic evolution: the price equation and the replicator/interactor distinction. Journal of Economic Methodology, v. 11, n. 2, p. $147-$ $173,2004$.

LALL, S.; TEUBAL, M. "Market-Stimulating” technology policies in developing countries: a framework with examples from East Asia. World Development, v. 26, n. 8, p. 1369-1385, 1998.

LEE, K. Schumpeterian analysis of economic catch-up: knowledge, path-creation, and the middle-income trap. Cambridge, MA: Cambridge University Press, 2013.

LUNDVALL, B. A. National system of innovation: towards a theory of innovation and interactive learning. London: Pinter Publishers, 1992.

Innovation system research. Where it came from and where it might go. Globelics, 2007 (Working Paper Series, 2007-01).

LUNDVALL, B. A .; JOSEPH, K. J.; CHAMINADE, C.; VANG, J. Handbook of innovation systems and developing countries: building domestic capabilities in a global setting. Cheltenham, UK: Edward Elgar Publishing, 2011.

LLERENA, P.; MATT, M. Innovation policy in a knowledge-based economy: theory and practice. Springer Science \& Business Media, 2006.

MALERBA, F.; ORSENIGO, L. Technological regimes and sectoral patterns of innovative activities. Industrial and Corporate Change, v. 6, n. 1, p. 83-118, 1997.

MARENGO, L.; DOSI, G. Division of labor, organizational coordination and market mechanisms in collective problem-solving. Journal of Economic Behavior \& Organization, v. 58, n. 2, p. 303-326, 2005.

METCALFE, J. S. Technology systems and technology policy in an evolutionary framework. Cambridge Journal of Economics, v. 19, n. 1, p. 25-46, 1995.

Dancing in the dark: la disputa sobre el concepto de competencia. Desarrollo Económico, v. 50, n. 197, p. 59-79, abr.-jun. 2010.

METCALFE, J. S.; FOSTER, J.; RAMLOGAN, R. Adaptive economic growth. Cambridge Journal of Economics, v. 30, n. 1, p. 7-32, 2006.

NELSON, R. National innovation systems: a comparative analysis. Oxford: Oxford University Press, 1993.

NELSON, R.; WINTER, S. An evolutionary theory of economic change. Cambridge, MA: The Belknap Press of Harvard University Press, 1982. 
O' HARA, P. A. Principle of circular and cumulative causation: Fusing Myrdalian and Kaldorian growth and development dynamics. Journal of Economic Issues, v. 42, n. 2, p. 375-387, 2008.

PAMPLONA, J. B.; CACCIAMALI, M. C. O paradoxo da abundância: recursos naturais e desenvolvimento na América Latina. Estudos Avançados, v. 31, n. 89, p. 251-270, 2017.

PERES, W. El lento retorno de las políticas industriales en América Latina y el Caribe. Revista de la Cepal, n. ${ }^{o}$ 88, p. 71-88, Abril 2006.

PÉREZ, C. Dinamismo tecnológico e inclusión social en América Latina: una estrategia de desarrollo productivo basada en los recursos naturales. Revista de la Cepal, n. 100, p. 123-145, 2010. . Réplica a los comentarios. Revista Econômica, v. 14, n. 2, p.73-81, 2012.

PÉREZ, Caldentey E. Una coyuntura propicia para reflexionar sobre los espacios para el debate y el diálogo entre el (neo) estructuralismo y las corrientes heterodoxas. Neoestructuralismo y corrientes heterodoxas en América Latina y el Caribe a inicios del siglo XXI. Santiago: Cepal, 2015. p. 33-91.

POSSAS, M. L. Estructuras de mercado em oligopólio. São Paulo: Hucitec, 1985.

ROBERT, V.; YOGUEL, G. Complexity paths in neo-Schumpeterian evolutionary economics, structural change and development policies. Structural Change and Economics Dynamics, v. 38, p. 3-14, 2016.

SAVIOTTI, P. P. Variety, growth and demand. Journal of Evolutionary Economics, v. 11, n. 1, p. 119-142, 2010.

SAVIOTTI, P. P.; PYKA, A. Economic development by the creation of new sectors. Journal of Evolutionary Economics, v. 14, n. 1, p. 1-35, 2004.

SETTERFIELD, M. Endogenous growth: a Kaldorian approach. 2010. Disponible en: <https://papers.ssrn.com/sol3/papers.cfm?abstract_id=1597944>.

SUZIGAN, W.; FURTADO, J. Política industrial e desenvolvimento. Revista de Economía Política, v. 26, n. 2, (102), p. 163-185, 2006.

TEDESCO, J. C. Industrialización y educación en la Argentina. Buenos Aires: Unesco/Cepal/ PNUD, Proyecto Desarrollo y Educación en América Latina y el Caribe, 1977.

WITT, U. Self-organization and economics - what is new? Structural Change and Economic Dynamics, v. 8, n. 3, p. 489-507, 1997.

YOGUEL, G.; BOSCHERINI, F. El desarrollo de las capacidades innovativas de las firmas y el rol del sistema territorial. Desarrollo Económico, v. 41, n. 161, p. 37-69, 2001. 